\title{
Laparoscopic ultrasound with radiofrequency ablation of hepatic tumors in cirrhotic patients
}

\author{
Ibrahim Abdelkader Salama, ${ }^{a} M D$; Enas Korayem, ${ }^{b} M D ; O s a m a$ El-Abd, ${ }^{b} M D$; \\ Ahmed El-Refaie, ${ }^{c} M D$ \\ a) Department of Surgery, National Liver Institute, Menouphyia University, Shibin \\ El kom, Egypt. \\ b) Department of Radiology, National Liver Institute, Menouphyia University, \\ Shibin El kom, Egypt. \\ c)Department of Pathology, National Liver Institute, Menouphyia University, \\ Shibin El kom, Egypt.
}

\begin{abstract}
Background: The optimal treatment for hepatocellular carcinoma (HCC) is surgical resection. However, only a small percentage of patients are operative candidates due to associated liver cirrhosis. Recent advances in laparoscopic ultrasound and laparoscopy have greatly improved the accuracy in detecting intrahepatic tumors nodules, many of which were missed by preoperative imaging modality.

Objective: Evaluation the safety and efficacy of laparoscopic radiofrequency ablation (RFA) guided with laparoscopic ultrasound in detection and treatment of liver tumors in patients with liver cirrhosis.

Methods: 72 patients with liver tumors (58 HCC, 9 metastatic adencarcinoma, 2 neoendocrine metastasis, 3 other metastasis) were submitted to laparoscopic RFA under laparoscopic ultrasound guidance. 44 patients (61.1\%) Child A and 28 patients (38.9\%) Child B. Patients with large tumor (>6 cm), portal vein thrombosis, or Child $C$ class were excluded from the study.

Results: Laparoscopic RFA were completed in all patients without any conversion rate. Laparoscopic ultrasound identified 19 new malignant lesions (18.4\%) in comparison with the result of preoperative imaging. A total of 103 hepatic focal lesions were treated by RFA (45 patients had one lesion, 23 patient had 2 lesions and 4 patients had 3 lesions). There was no mortality. Morbidity occurred in 4 patients (5.5\%) 2 patients had liver abscesses, one patient had pleural effusion and one patient had postoperative bleeding necessitating blood transfusion and surgery. After a mean follow up of $14.3 \pm 11.6$ months, a complete response with $100 \%$ necrosis was achieved in 93 out of 103 lesions(90.3\%). 3 lesions (2.9\%) showed local recurrences, 5 lesions (4.8\%) showed remote recurrences, and 2 lesions (1.9\%) showed both local and remote recurrences.

Conclusion: Laparoscopic RFA guided with laparoscopic ultrasound is an excellent use of existing technology in improvement of safety and efficacy of detection and treatment of intrahepatic tumors in patients with liver cirrhosis.
\end{abstract}

Key words: Laparoscopy - Radiofrequency ablation (RFA) - Liver tumors.

\section{Introduction:}

Hepatocellular carcinoma (HCC) is a common cause of death in patients with cirrhosis. The optimal treatment for hepatocellular carcinoma (HCC) is surgical resection. However, only a small percentage of patients are operative candidates due to associated liver cirrhosis. ${ }^{1}$
In most patients with $\mathrm{HCC}$, treatment options are limited by liver dysfunction caused by chronic inflammation and cirrhosis. Although complete surgical resection of HCC offers the best chance of long-term survival, cirrhosis may limit the amount of parenchymal resection that will be tolerated and increases the risk of postoperative liver failure and death. ${ }^{2,3}$ 
Systemic or regional chemotherapy is at least palliative in a small subset of patients (although rarely patients have sufficient tumor down staging to convert an unresectable to a resectable lesion), but at the cost of significant side effects and a reduced quality of life. ${ }^{1}$

In most patients with cirrhosis and HCC confined to the liver, resection is not safe, and local tumor ablation therapies are considered as alternative treatment options.

For centuries, it has been recognized that heat is an effective means of destroying tissues. Once cells are heated above $50^{\circ} \mathrm{C}$, cell membranes melt and fuse, and with continued heating, proteins denaturation and irreversible cell death occur. ${ }^{4,5}$

Radiofrequency ablation (RFA) is a thermal treatment technique designed to produce localized tumor destruction by heating tumor tissue to temperatures that exceed $50^{\circ} \mathrm{C}$. When tumor cells are heated to temperatures above $45^{\circ} \mathrm{C}$ to $50^{\circ} \mathrm{C}$ for more than 3 minutes intracellular protein denaturation and melting of lipid bilayers results in direct tumor cell death. 6

Imaging plays a crucial role in the followup of hepatic tumors treated with RF ablation, as it is the means by which local treatment efficacy, recurrent disease, and some of therapy-induced complications are evaluated. Multi-phase helical computed tomography (CT) has been regarded as the method of choice in the evaluation of patients with hepatocellular carcinoma (HCC) treated by non surgical procedure. $^{7}$

Although the RFA procedure can be performed either via laparotomy or percutaneously, there is little information on its optimal approach. There are few data on laparoscopic approach in the treatment of patients with unresectable hepatic malignancies in cirrhotic patient. ${ }^{8}$

Recent advances in laparoscopic ultrasound have greatly improved the accuracy in detecting intrahepatic HCC nodules many were missed by radiology. Besides the detection of unsuspected intra-abdominal metastasis with laparoscopic ultrasound, the laparoscopic approach may have other potential benefits such as direct visual control of the RFA procedure; exposure and isolation of the liver from surrounding tissues; the ability to perform a Pringle maneuver; handling of intraoperative bleeding after biopsy or other complications; ablation of several lesions during one operation; the possibility of repeating laparoscopic RFA with low morbidity and minimal surgical trauma and postoperative pain; fast recovery time and shorter hospital stay.

The objective of this study was to evaluate the safety and efficacy of laparoscopic radiofrequency ablation (RFA) guided with laparoscopic ultrasound in detecting and treatment of liver tumors in patients with liver cirrhosis.

\section{Patients and methods:}

Seventy-two patients (49 males and 23 females; mean age 47 years (range $35-67$ years) with liver tumors in cirrhotic liver were submitted to laparoscopic RFA under laparoscopic ultrasound guidance. All cases were discussed in details at a multidisciplinary meeting and were approved by the institutional review board committee at National Liver Institute, Menouphyia University to study the safety and efficacy of laparoscopic radiofrequency thermal ablation. Informed consent was taken from all cases.

Selection criteria, which varied by tumor type, included unresectable primary or metastatic liver tumors and patient Child A or $\mathrm{B}$ class of liver functions. Exclusion criteria include Child C class of liver function, patient with large tumor $(>6 \mathrm{~cm})$ and patient with portal vein thrombosis.

Preoperative evaluation included history and physical examination; serum laboratory tests consisting of a complete blood picture, platelets, coagulation profile, virology, renal profile, electrolytes, liver functions \& serum tumor markers e.g. alpha fetoprotein (AFP).

Diagnostic imaging consisted of triphasic spiral computed tomography (CT) and/or magnetic resonance imaging (MRI) scan of the abdomen and pelvis and pulmonary Roentgenography.

All patients had histological confirmation of HCC \& or metastatic lesion from intraopererative biopsy or from prior CT or ultrasound- guided percutaneous fine needle aspiration. 


\section{Laparoscopic RFA procedure:}

Laparoscopic RFA procedure was performed with the patient under general anesthesia and positioned supine on the operating table. We prefer to use an optical access trocar (Optiveiw; Ethicon Endosurg, Inc) to enter the abdominal cavity. The procedure is performed using two $11-\mathrm{mm}$ trocars placed beneath the right costal margins and additional trocars were placed if needed to take down adhesions. One trocar was used for a $45^{\circ} \mathrm{C}$ laparoscope and the other port for 8666-RF laparoscopic transducer with fourway tip and built-in biopsy guide (B-K Medical, Mileparken 34, DK-2730 Herlev, Denmark).

The procedure started by a diagnostic laparoscopy to rule out any extra hepatic disease then the entire liver parenchyma was examined. All tumors within the liver were measured and assigned an anatomical liver segment by using the Couinaud's classification of the liver anatomy and color flow imaging is performed to assess the vascularity of the lesions. Representative tumors were then biopsied by using biopsy guide channel in laparoscopic ultrasound transducer for histological confirmation under ultrasound guidance.

After the lesions have been identified and measured, radiofrequency ablation was performed with a Rita Medical System Model 3 four prong $3-\mathrm{cm}$ in diameter thermal ablation catheter (Rita Medical System, Mountain view, CA).

Radiofrequency thermal ablation catheter is placed percutaneously into the lesion and the prongs are deployed but in most cases we used the biopsy guide channel to deploy the radiofrequency ablation with use of flexible radiofrequency needle fit with the laparoscopic ultrasound. A rim of normal liver parenchyma is ablated also to enable achievement of margins around the tumor, analogous to resection. All time the procedure was guided with laparoscopic ultrasound to precise and locate the ablated lesion and to detect any new lesion Figure(1,2).

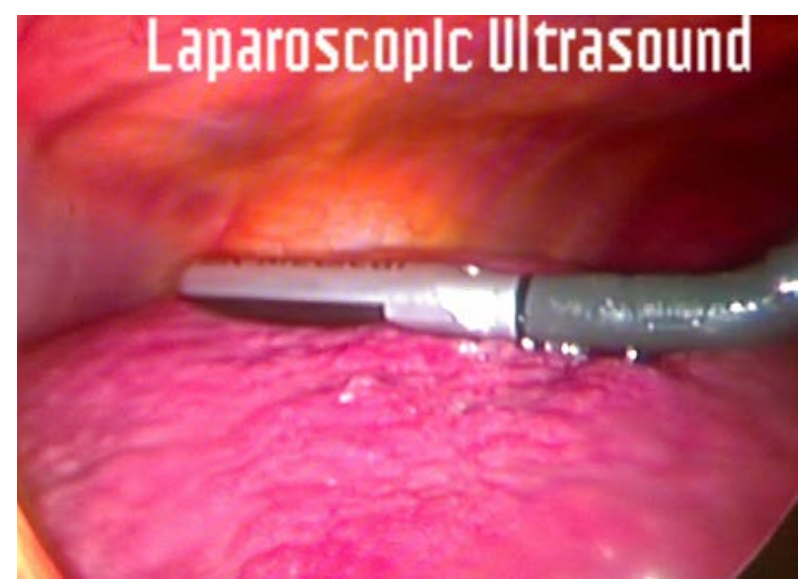

Figure (1): Laparoscopic ultrasound.

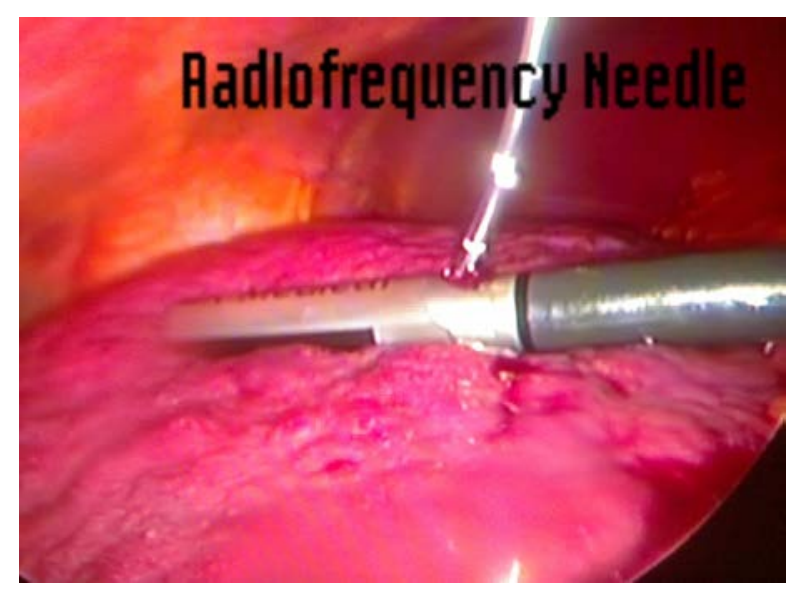

Figure (2): Radiofrequency needle ablation through laparoscopic ultrasound.

outgassing of dissolved nitrogen within the tissues), which appear at the ablated volume. The nitrogen resorbs after approximately 10 minutes. The loss of blood flow within the tumor, on color Doppler analysis, was also used particularly in highly vascular tumors, to confirm complete ablation. Figure(3) 

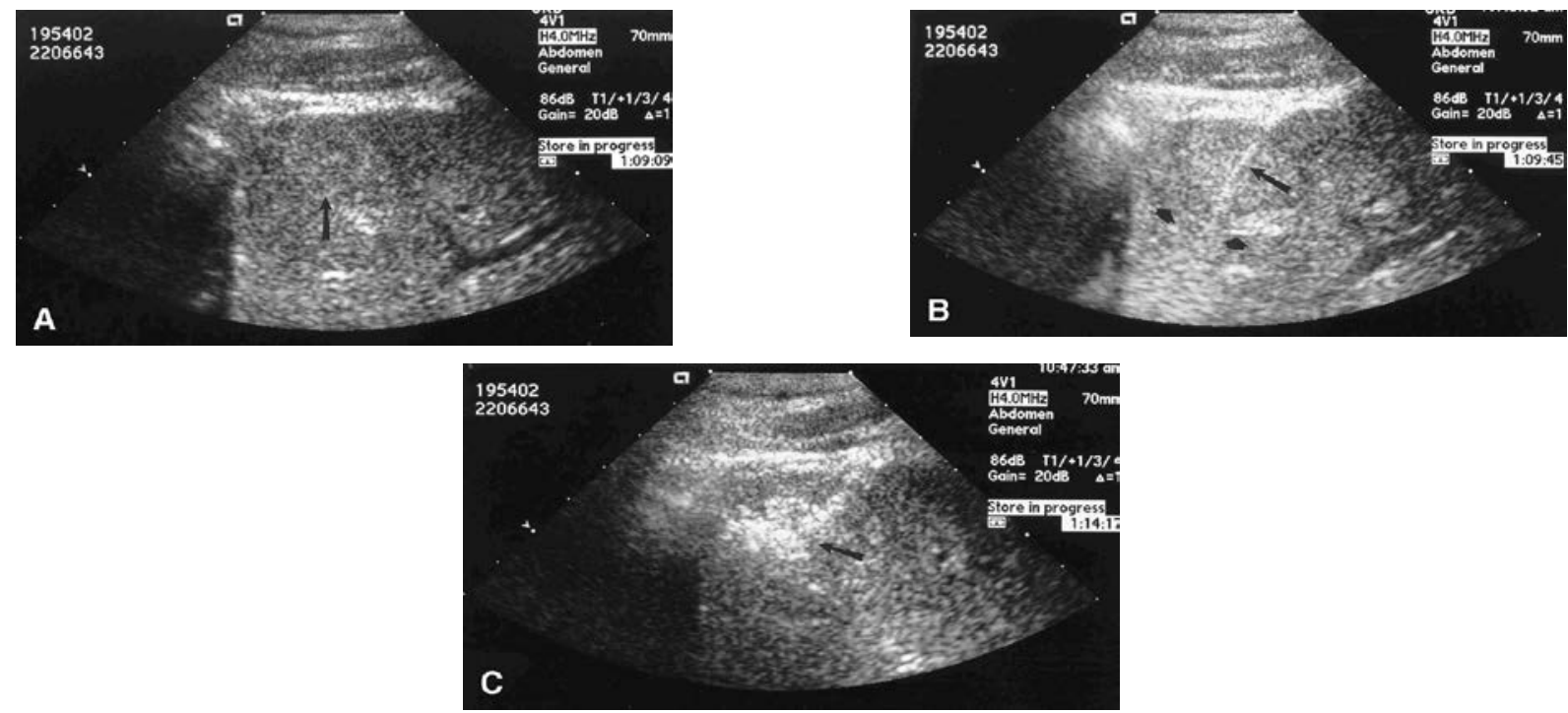

Figure (3): (A) Laparoscopic ultrasonography demonstrating a solitary hepatocellular carcinoma (arrow). (B) The radiofrequency ablation needle (long arrow) has been placed and the multiple arrays opened (Short arrows) at the deep interface between tumor and hepatic parenchyma. (C) The area of tumor and liver treated with radiofrequency ablation becomes hyperechoic on ultrasound (arrow). The multiple arrays are subsequently retracted back into the needle electrode sheath, the needle is pulled back approximately $2 \mathrm{~cm}$, and then the array is redeployed to complete the radiofrequency ablation treatment of the tumor.

In most cases, patients are monitored overnight and discharged to go home the morning after surgery.

\section{Triple-phase helical CT study:}

CT was performed within 2-5 days (initial), one month after the procedure, and then every 3 months. All patients underwent non enhanced and contrast enhanced triple-phase helical CT. A total of $120 \mathrm{ml}$ non ionic contrast medium was administrated at a rate of $3 \mathrm{ml} / \mathrm{sec}$ with an automatic power injector. Three spiral CT scans were obtained during the hepatic arterial phase, the portal venous phase, and the equilibrium phase at 30,60 , and 180 seconds respectively after initiation of the injection. The images were obtained in craniocaudal direction with $7 \mathrm{~mm}$ collimation and $7 \mathrm{~mm} / \mathrm{sec}$ table speed. Each spiral acquisition through the liver was accomplished during a breath hold.

\section{Image analysis:}

On unenhanced and triple-phase contrastenhanced CT scans, the size and shape of the treated area were evaluated at each imaging follow up on CT images, in addition to the presence of peripheral nodular enhancement (residual viable tumor), peripheral rim enhancement (reactive hyperemia), vascular changes, and complications. We also evaluated findings in the abdomen or lower chest that could be related to the ablation procedure.

All contrast-enhanced foci on 4 months and after follow up CT images with nodular pattern or irregular thickening of the boundaries of the ablated area were considered as suspicious for local tumor recurrence. The appearance of new intrahepatic lesions (remote recurrence) was also assessed. A non enhancing area of low attenuation that did not demonstrate contrast enhancement within the boundaries of the ablated areas on follow up CT images were considered as successfully treated tumor. ${ }^{9}$ The CT findings obtained after ablation were compared with those obtained before ablation to ensure that the changes were not present before RF ablation.

\section{Statistical analysis:}

Statistical analysis was estimated by KaplanMeier analysis. Difference in survival was analyzed using the long. Rank test. Differences in tumor recurrence rates were analyzed using the Fisher exact test and 2 sided $(\mathrm{Chi})^{2}$ test. Differences were considered to be statistically significant when the $\mathrm{P}$ value was $<0.05$. 


\section{Results:}

Laparoscopic radiofrequency ablation procedure was completed in all patients without any conversion rate. 44 patients $(61.1 \%)$ were Child-Turcotte-Pugh A class of liver function and 28 patients (38.9\%) were Child-TurcottePugh B class of liver function.

The tumor histolopathology results were $58 \mathrm{HCC}(80 \%), 9$ metastatic adenocarcinoma (12.5\%), 2 neoendocrine metastasis $(2.7 \%)$ and 3 other metastasis (4\%).

Laparoscopic ultrasound identified 19 new malignant lesions (18.4\%) in comparison with the result of preoperative imaging.

RFA was used to ablate a total 103 ultrasonographically detected tumor nodules in 72 patients. RFA was used to treat one tumor in 45 patients $(62.5 \%)$, two lesions in 23 patients $(31.9 \%)$ and three lesions in four patients $(5.5 \%)$.

Size of the tumor ablated varied from $1 \mathrm{~cm}$ to $2 \mathrm{~cm}$ in 51 patients (49.9\%), $2 \mathrm{~cm}$ to $4 \mathrm{~cm}$ in 36 patients $(35 \%)$ and $4 \mathrm{~cm}$ to $6 \mathrm{~cm}$ in 16 patients (15.5\%) Table(1).

The laparoscopic ultrasound detected exactly the site of the lesions in liver segments (segment I (17 lesions), segment II (12 lesions), segment III (8 lesions), segment IV (20 lesions), segment V (14 lesions), segment VI (7 lesions), segment VII (9 lesions) and segment VIII (16 lesions). Most of the lesions were in a central segment of the liver.

Length of hospital stay averaged 1.2 \pm 0.6 days.

\section{Table (1): Characteristics of patients and hepatic focal lesions treated with RF ablation.}

\begin{tabular}{|c|c|c|}
\hline Parameter & No. & Percentage $\%$ \\
\hline $\begin{array}{l}\text { Patients }(\mathbf{n = 7 2}) \\
\text { Male } \\
\text { Female }\end{array}$ & $\begin{array}{l}49 \\
23\end{array}$ & $\begin{array}{l}68 \% \\
32 \%\end{array}$ \\
\hline $\begin{array}{l}\text { Child-Pugh cirrhosis } \\
\text { Class A } \\
\text { Class B }\end{array}$ & $\begin{array}{l}44 \\
28\end{array}$ & $\begin{array}{l}61 \% \\
39 \%\end{array}$ \\
\hline $\begin{array}{l}\text { Tumor pathology } \\
\text { HCC } \\
\text { Metastatic adenocarcinoma } \\
\text { Neuroendocrine metastasis } \\
\text { Other metastasis }\end{array}$ & $\begin{array}{c}58 \\
9 \\
2 \\
3\end{array}$ & $\begin{array}{l}80.5 \% \\
12.5 \% \\
2.7 \% \\
4 \%\end{array}$ \\
\hline $\begin{array}{l}\text { Tumor multiplicity } \\
\text { Solitary } \\
2 \text { lesions } \\
3 \text { lesions }\end{array}$ & $\begin{array}{c}45 \\
23 \\
4\end{array}$ & $\begin{array}{l}62 \% \\
32 \% \\
5.5 \%\end{array}$ \\
\hline Hepatic focal lesions $(n=103) *$ & & \\
\hline $\begin{array}{l}\text { Tumor size } \\
1-2 \mathrm{~cm} \\
2-4 \mathrm{~cm} \\
4-6 \mathrm{~cm}\end{array}$ & $\begin{array}{l}51 \\
36 \\
16\end{array}$ & $\begin{array}{c}49 \% \\
35 \% \\
15.5 \%\end{array}$ \\
\hline $\begin{array}{l}\text { Tumor locations } \\
\text { Seg I/II } \\
\text { Seg III/IV } \\
\text { Seg V/VI } \\
\text { Seg VII/VIII }\end{array}$ & $\begin{array}{l}17 / 12 \\
8 / 20 \\
14 / 7 \\
9 / 16\end{array}$ & $\begin{array}{l}16.5 / 11.6 \% \\
7.7 / 19.4 \% \\
13.6 / 6.8 \% \\
8.7 / 15.5 \%\end{array}$ \\
\hline
\end{tabular}

*19 new malignant lesions that were not diagnosed by preoperative imaging had been detected by laparoscopic ultrasound 
Postoperative laboratory studies demonstrated a mild elevation in the white blood count, but it remained in the normal range. Platelet count showed a minimal drop on postoperative day 1 , but returned to normal range within a week. International normalized ratio (INR) showed no fluctuation. Serum alanine transaminases and aspartate transaminases typically demonstrated a 10 fold increase on the day of surgery, but they returned to near normal levels within 1 week. Alkaline phosphatase showed a mild elevation that peaked later than the transaminases, but it quickly returned to normal range. Serum bilirubin also demonstrated slight elevation on postoperative day 1 but usually did not exceed the upper limit of normal.
Serum AFP values were elevated ( $>5.0$ $\mathrm{ng} / \mathrm{ml})$ in 66 patients $(91.6 \%)$ at the time of diagnosis. Serum AFP levels were remeasured 1 month after RFA treatment. AFP values after RFA in all patients $(4.5 \pm 0.4 \mathrm{ng} / \mathrm{ml})$ were significantly lower than the AFP measurement before RFA treatment $(118.4 \pm 24.2 \mathrm{ng} / \mathrm{ml}, \mathrm{P}<$ $0.01)$.

\section{Morbidity and mortality:}

There was no mortality. Morbidity occurred in 4 patients $(5.5 \%) ; 2$ patients had liver abscesses treated by percutaneous drainage, one patient had pleural effusion and one patient had postoperative bleeding necessitating blood transfusion and surgery Table(2).

Table (2): Postoperative morbidity n=4 (5.5\%).

\begin{tabular}{|l|lc|}
\hline Complications & No. & $\%$ \\
\hline Liver abscesses & 2 & $2.7 \%$ \\
Pleural effusion & 1 & $1.4 \%$ \\
Bleeding & 1 & $1.4 \%$ \\
\hline
\end{tabular}

At initial and 1 month follow-up CT images, a complete response with $100 \%$ necrosis was achieved in all focal lesions ( $\mathrm{n}=103)$ Figure(4). However, within mean follow up of $14.3 \pm 11.6$ months Table(3), 3 lesions (2.9\%) showed local recurrences (enhancing tissue around the treated nodule) Figure(5), 5 lesions (4.8\%) showed remote recurrences, and 2 lesions $(1.9 \%)$ showed both local and remote recurrences Figure(6).

Table (3): Types of intrahepatic recurrences on helical CT images after RF therapy $n=10$ (9.7\%).

\begin{tabular}{|l|c|c|c|}
\hline Duration of CT follow up & Types of recurrences & No & Percentage \% \\
\hline 4 months & $\begin{array}{c}\text { Local } \\
\text { Local and remote }\end{array}$ & $\begin{array}{l}2 \\
2\end{array}$ & $\begin{array}{l}1.9 \% \\
1.9 \%\end{array}$ \\
\hline 7 months & Local & 1 & $1 \%$ \\
& Remote & 3 & $2.9 \%$ \\
\hline 10 months & Remote & 1 & $1 \%$ \\
\hline 13 months & Remote & 1 & $1 \%$ \\
\hline 16 months & - & - & - \\
\hline
\end{tabular}



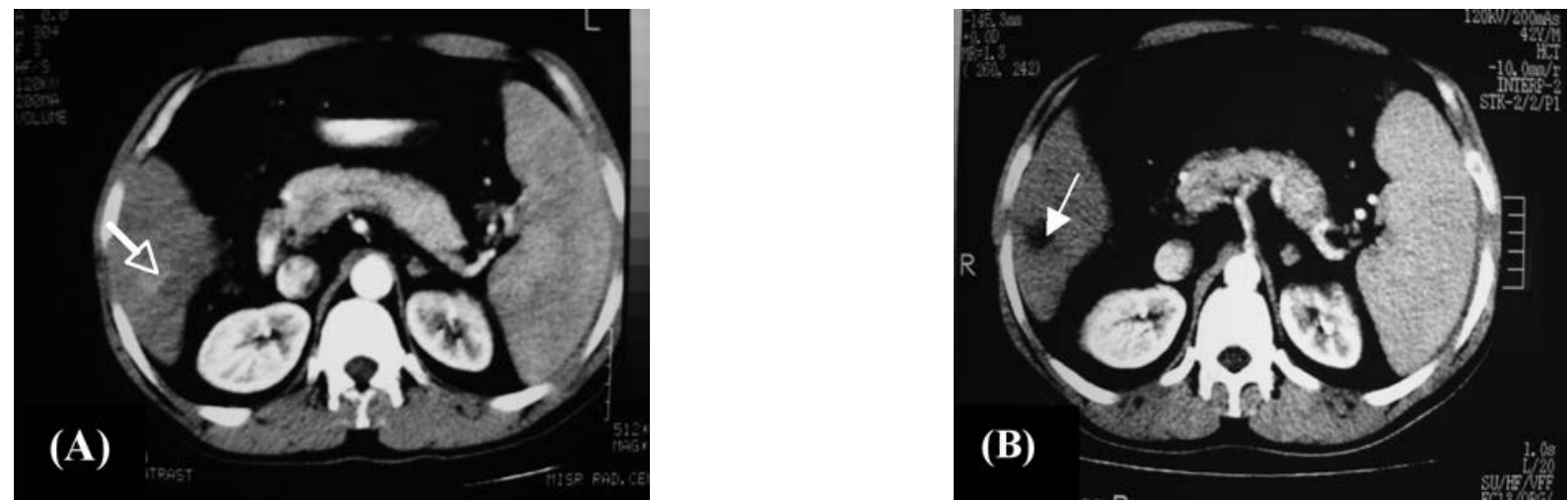

Figure (4): Complete ablation of hepatic nodule. (A) Contrast enhanced axial helical CT scan obtained during the arterial phase before RF ablation shows an enhancing hepatic nodule at segment VI of the right hepatic lobe (arrow). (B) Contrast enhanced axial helical $C T$ scan obtained 4 days after RF ablation shows a hypoattenuating-ablated area with absence of contrast enhancement (arrow).
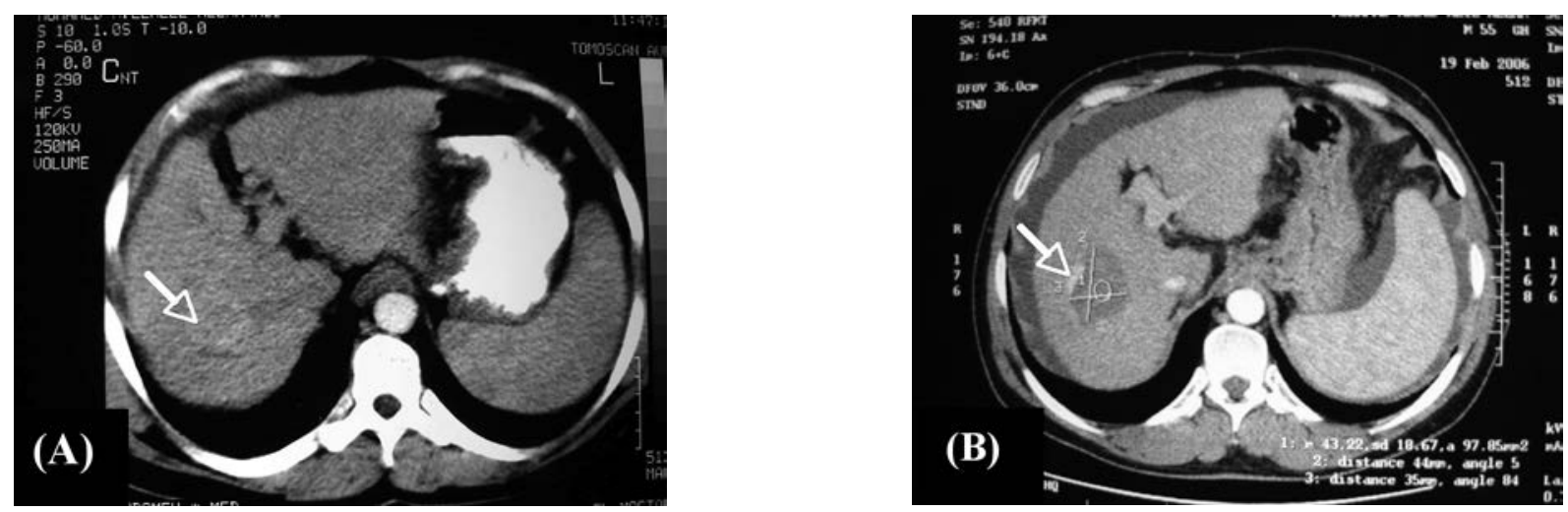

Figure (5): Local recurrence of hepatic nodule after RF ablation. (A) Contrast enhanced axial helical CT scan obtained during the arterial phase before RF ablation shows an enhancing hepatic nodule at segment VII of the right hepatic lobe (arrow). (B) At 7-months follow-up arterial phase contrast enhanced CT image shows an enhancing nodule abutting the $R F$ treated area (arrow).

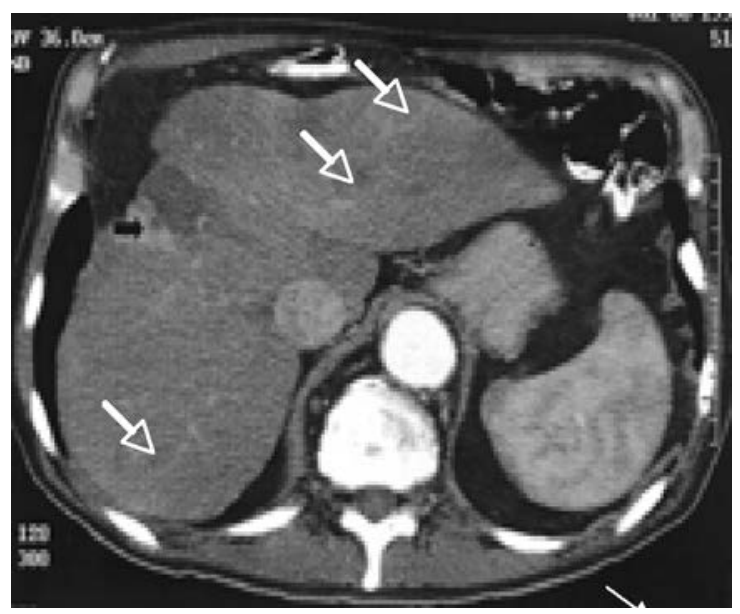

Figure (6): Local and remote recurrences of hepatic nodule after RF ablation. At 4 months follow up arterial phase contrast enhanced CT image shows an enhancing nodule abutting the $R F$ treated area at segment IV (black arrow) other new nodules that show peripheral enhancement had been developed remote from the previously ablated lesion are seen at the right and left hepatic lobes of the liver (white arrows). 


\section{Discussion:}

The use of heat to treat human neoplasm is not a recent development. The first recorded descriptions of therapeutic thermal tumor ablation came from the Edwin Smith and Ebers surgical papyri, describing the use of cautery with heated implements or oils to destroy tumors. The practices described in these papyri date to the 2nd Egyptian dynasty and the physician Imhotop approximately 5,000 years ago. ${ }^{10}$

The international application of an electrical current to produce thermal ablation of a tumor dates to the early 20th century. In 1909, Pozzi, coined the term "Fulguration" in a description of curative treatment of superficial skin cancers with a high frequency. ${ }^{11}$ Doyen subsequently applied a ground plate to patients to improve electrofulguration of tumor. Electrocautry devices are now routinely used by surgeons to achieve haemstasis during surgical procedures. ${ }^{12}$

Patients with unresectable hepatic malignancies have a dismal prognosis. Liver resection may not be indicated for patients with hepatic cancer in both lobes or with involvement of a major vascular structure in which resection would result in postoperative liver failure because of insufficient residual functional liver parenchyma. Therefore the treatment modalities and approaches for patients with hepatic cancer should not only aim at potential cure or control of hepatic disease but also be minimally invasive.

Radiofrequency ablation (RFA) is a safe and effective treatment modality to achieve tumor destruction in patients with unresctable hepatic malignancies. ${ }^{13}$

Radiofrequency ablation (RFA) is one of the recent treatment modalities that have increased the size of the pool of patients that may benefit from aggressive liver directed therapy. Unfortunately, most patients with primary or metastatic liver malignancies are not candidates for resection because of tumor number, pattern of spread throughout the liver, location near key vascular structures precluding margin-negative resections, or inadequate functional hepatic reserve revealed to coexistent cirrhosis. Local destruction of an intrahepatic tumor by RFA can be used to treat some patients with unresectable disease, thus providing them with hope on long term disease control. ${ }^{13}$

This study demonstrates that radiofrequency thermal ablation can be successfully performed laparoscopically in a variety of tumor histologies (primary \& metastatic liver malignancies) in cirrhotic patients. The ability to see and treat lesions at the dome of the liver, and to treat lesions that were peripheral in the liver or in proximity to other organs, increases the flexibility of the procedure over percutaneous approach while remaining minimally invasive. The placement of the needle in the lesions was extremely accurate by using laparoscopic ultrasonography, with most of the lesions appearing almost entirely.

One of the greatest advantages of a laparoscopic approach is that, similar to open surgery, there is the ability to target lesions precisely with intraoperative ultrasonography. However, the minimally invasive approach reduces hospital stay and hence cost, reduces postoperative narcotic requirements and reduces post discharge convalescence. ${ }^{14}$

For both $\mathrm{HCC}$ and metastatic liver tumor patients in M.D.Andrson /G.Pascale series, serum liver function tests (e.g. alanine aminotransferase, aspartate aminotransferase and gamma glutamyltransferase) were elevated 2- to 3 folds above baseline values immediately following the procedure, but for most patients, these values returned to baseline levels within 7 days, and for all patients the values were normal within 1 month after the procedure. ${ }^{15}$

Our series showed that a postoperative rise in liver function tests is expected and reflects the underlying liver injury response to RFA. The most dramatic elevation is seen in the liver transaminases with AST and ALT showing a 10 -fold elevation. The transamionases return to baseline within a week. Serum alkaline phosphates demonstrate an elevation in a week then return to normal, the degree of postablation elevation is dependent on the amount of normal liver parenchyma subjected to RFA. So, we were not alarmed by the early dramatic rises in AST and ALT levels because they result from destruction of a liver parenchyma margin surrounding the tumor and do not indicate pending complication. 
RFA can be used to treat patients with hepatic tumor in a location that precludes a margin-negative hepatic resection, such as a tumor rested between the inferior vena cava (IVC) and the entrance of the three hepatic veins into the IVC. 16

A laparoscopic approach offers the advantage of laparoscopic ultrasonography, which provides better resolution compared with transcutaneous ultrasonography of the number and location of liver tumors and a survey of the peritoneal cavity to exclude the presence of extra hepatic disease. Using laparoscopic ultrasound guidance, the RFA needle electrode is advanced percutaneously or through laparoscopic ultrasound side channel into the target tumors for treatment. The laparoscopic ultrasound permits more precise positioning of the RFA needle multiple arrays near major blood vessels. A laparoscopic approach may be ideal for patients with no prior history of extensive abdominal operations and one or two tumors $<4.0 \mathrm{~cm}$ in diameter located centrally in the liver near major intrahepatic blood vessels. ${ }^{17}$ Also due to the laparoscopic ultrasound guidance there were 19 new malignant lesions that were discovered in 7 patients not diagnosed by work up done preoperatively in our study.

We successfully treated tumors of almost all the liver segments mainly in central segment locations of the liver which is challenge in surgical resection (17 lesions in segment I, 20 lesions in segment IV and 16 lesions in segment VIII ).

The only area of the liver to avoid when treating a tumor with RFA is the hilar plate where the portal vein and hepatic arterial branches enter the liver although these blood vessels can tolerate the RFA treatment. The large bile ducts coursing with them do not tolerate heat and biliary fistula or stricture would occur after RFA. RFA is ideally suited to treat small HCC in cirrhotic patients, who may not be candidates for resection based on the severity of their liver dysfunction. ${ }^{18}$

For patients with hepatic tumors that are not amenable to resection (for whatever reason) RFA provides good local control and improve survival. The local recurrence rate (defined as a recurrence at the ablation zone) ranged from
$1.8 \%$ to $26.6 \%$. Certainly, proper patient selection is a key success factor. ${ }^{19}$

Some studies have also shown that the approach chosen for delivery of RFA (open, laparoscopic or percutaneous) may impact local recurrence. Kuvshinoff (2002) 20 \& Scaife $(2003)^{21}$ showed lower rates of local recurrence for open and laparoscopic RFA versus a percutaneous approach. The increased access to the liver and use of intraoperative ultrasound are likely the main reasons for lower local recurrence rates for operative RFA. It should be mentioned that in our study all RF treated nodules were completely ablated with no residual tumor. Curely et al., 200022 supported our results. Post treatment peripheral enhanced ring in the site of the treated tumor remains an intriguing observation. It may be caused by post necrotic edema, new vascularization with microscopic arteriovenous shunting, or residual viable tumor. Therefore to avoid difficulties in imaging interpretation, dynamic CT scans should be obtained 3-4 days after the RF interstitial thermal ablation because the size of necrosis at the maximum and the vascular neogenesis is poor. ${ }^{23}$

The absence of contrast enhancement in the ablated lesion at short-term follow up CT within 3 months after treatment does not always indicate successful treatment, as later follow up studies can demonstrate tumor regrowth at the periphery of the ablated lesions. ${ }^{24}$

After a mean follow up $14.3 \pm 11.6$ months recurrence rate in our series: 3 (2.9\%) focal lesions showed local recurrence which are much lower than the other studies, $5(4.8 \%)$ focal lesions showed remote recurrences, and $2(1.9 \%)$ focal lesions showed both local and remote recurrences. Ablation site recurrence rates had been reported by many. ${ }^{25}$ Local regrowths were always depicted at the periphery of the treated area either as irregular thickening of one margin of the treated area, or a new tumor nodule. These peripheral locations of treatment failures could be explained by lower energy deposition, and reduced heating that was remote from the needle electrode. Furthermore, tissue perfusion lowers heat accumulation due to cooling, and this phenomenon is even more marked in tissue in contact with large vessels. ${ }^{26}$ 
The complication rate associated with RFA is very low, ranging from $2.4 \%$ to $27 \%$ in several trials in a large review. $27,28,29,30-31$

Scaife $(2003)^{21}$ reported an overall mortality rate of $0.5 \%$, a major complication rate of $2 \%$ and a minor complication rate of $6 \%$ by combining over 1300 patients from 18 different studies. The complications following RFA include wound infections, intraabdominal abscess, renal failure, hepatic abscess, biliary injuries, pleural effusion and minor hemorrhage.

Other CT findings related to the procedure have been reported: Perihepatic effusion, segmental biliary duct dilatation and local atrophy, arterioportal fistula, subcapsular collection and plural effusion. ${ }^{32}$ Moreover, intralesional air was found in Lim et al., $2001^{33}$ studies at immediate follow up CT, and disappeared at 1-month follow up CT. It is believed that air without an air-fluid level may have been introduced along the insertion path of the needle or may have resulted from tissue necrosis. ${ }^{34}$

In present study the morbidity occurred in 4 patients (5.5\%), 2 patients had liver abscesses who were treated by percutaneous drainage, one patient had pleural effusion and one had internal hemorrhage necessitating blood transfusion and surgery. Our study had a lower rate of complications than the other studies.

We found a relatively low complication rate after RFA because of careful treatment planning. We have avoided thermal injury to adjacent structures such as diaphragm or bowel by using laparoscopic approach to retract adjacent structures away from tumors near the liver capsule.

There is little debate on the improved recovery time from a laparoscopic operation versus a traditional open surgical procedure. Smaller incisions, less body wall and rib retraction and an improved cytokine response all favor the laparoscopic approach. Laparoscopic approach is safe, effective, and of potential benefit to the patient in terms of morbidity and recovery. Most of patients who are being treated with RFA are candidates for a laparoscopic approach. Laparoscopic probe placement and tumor ablation, and the laparoscopic approach aids with protection of the other viscera (pneumoperitoneum and retraction).
In conclusion, laparoscopic RFA guided with laparoscopic ultrasound is an excellent use of existing technology in improvement of safety and efficacy of detection and treatment of intrahepatic tumors in patients with liver cirrhosis.

\section{References:}

1- Watkins KT, Curley SA: Liver and bile ducts. In: Clinical Oncology. Abebff M.D, Armitage JO, Lichter AS, Niederhuber JE (Editors). New York: Churchill Livingstone (Publishers); 2nd ed. 2000; p.1681-1748.

2- Fan ST, Ng IOL, Poon RTP: Hepatectomy for hepatocellular carcinoma. The surgeon role in long-term survival. Arch Surg 1999; 134: 1124-1130.

3- Mazziotti A, Grazi GL, Cavallari A: Surgical treatment of hepatocelluar carcinoma in cirrhosis; a western experience. HepatoGastroenterology 1998; 45: 1281-1287.

4- Lounberry W, Gold Schmidt V, Linke C: The early histologic changes following electrocoagulation. Gastrointestinal Endoscopy 1995; 41: 68-70.

5- Mc Gahan J, Brock J, Teslak H: Hepatic ablation with use of radiofrequency electrocautery in the animal model. $J$ Vas Interv Radiol 1992; 3: 391-397.

6- Rossi S, Fornari F, PatiesC, Buscarini L: Thermal lesions induced by $480-\mathrm{KHZ}$ localized current field in Guina pig and in pig livers. Tumori 1990; 76: 54-57.

7- Choi D, Lim HK, Kim SH, et al: Hepatocellular carcinoma treated with percutaneous radio-freqency ablation: Usefulness of power doppler US with a microbubble contrast agent in evaluating therapeutic response-preliminary results. Radiology 2000; 217: 558-563. 8- Siperstein A, Garland A, Engle K, Rogers S, Berber E, String A, Foroutuni A, Ryan $\mathrm{T}$ : Laparoscopic radiofrequency ablation of primary and metastatic liver tumors: Technical consideration. Surg Endoscopy 2000; 14: 400-405.

9- Solbiati L, Ierace T, Goldberg SN, et al: Percutaneous US-guided radio-frequency tissue ablation of liver metastases: Treatment and follow-up in 16 patients. Radiology 1997; 202: 195-203. 
10-Breasted IH: The Edwin Smith Surgical Papyrus. Chicago: Chicago University Presses 1930; 54.

11-Kelly HA, Ward GE: Electro surgery. Philadelphia: WB Saunders (Publishers). 1932; p. 1-9.

12-Bovie GRM: The man and the machine. Ann Plast Surg 1979; 2: 135-153.

13-Curley SA, Izzo F: Laparoscopic radiofrequency. Ann Surg Oncology 2000; 7: 78-79.

14-Siperstein A, Garland A, Engle K, Rogers S, Berber E, Foroutani A, et al: Local recurrence after laparoscopic radiofreuency thermal ablation of hepatic tumors. Ann Surg Oncology 2000; 7: 106-113.

15-Curley SA: Radiofrequency ablation of malignant liver tumors. The Oncologist 2001; 6: 14-23.

16-Curley SA, Davidson B S, Fleming RY, et al: laparoscopically guided bipolar radiofrequency ablation of areas of procaine liver. Surg Endoscopy 1997; 11: 729-733.

17-Siperstein AE, Rogers SJ, Hansen PD, Gitomirsky A: Laparoscopic thermal ablation of hepatic neuroendocrine tumor metastasis. Surgery 1997; 122: 1147-1154.

18-Curly SA, Izzo, Ellis LM, Nicolas Vauthey J, Vallone P: Radiofrequency ablation of hepatocellular cancer in 110 patients with cirrhosis. Ann Surg 2000; 232: 381-391.

19-Machi J, Uchida S, Sumidak, Limm WM, Hundachi S A, Oishi AJ, Furumoto NL, Oishi RH: Ultrasound-guided radiofrequency thermal ablation of liver tumors: Percutaneous, laparoscopic and open surgical approaches. J Gastrointes Surg 2001; 5: 477-489.

20-Kuvshinoff BW, Ota DM: Radiofrequency ablation of liver tumors: Influence of technique and tumor size. Surgery 2002; 132: 605-611.

21-Scaife CL, Curley SA: Complication, local recurrence and survival rates after radiofrequency ablation for hepatic malignancies. Surg Oncol Clin N Am 2003; 12: 243-255.

22-Curely SA, Izzo F, Ellis LM, et al: Radiofrequency ablation of hepatocellular cancer in 110 patients with cirrhosis. Ann Surg 2000; 232:1-19.
23-Rossi S, Stasi MD, Buscarini E, et al: Percutaneous RF interstitial thermal ablation in the treatment of hepatic cancer. AJR 1996; 167: 759-768.

24-Gazelle GS, Goldbeg SN, Solbiati L, et al: Tumor ablation with radio-frequency energy. Radiology 2000; 217: 633-646.

25-Bilchik AJ, Wood TF, Allegra D, et al: Cryosurgical ablation and radiofrequency ablation for unresectable hepatic malignant neoplasms: Proposed algorithm. Arch Surg 2000; 135: 657-662.

26-Dromain C, Baere I, Elias D, et al: Hepatic tumors treated with percutaneous radiofrequency ablation; CT and MR imaging follow-up. Radiology 2002; 223: 255-262.

27-Curley SA, Izzo F, Delrio P, Ellis LM, Granchi J, Vallone P, Fiore F, Pignata S, Daniele B, Cremona F: Radiofrequency ablation of unresectable primary and metastatic hepatic malignancies: Results in 123 patients. Ann Surg 1999; 230: 1-826.

28-Wong SL, Edwards MJ, Choa C, Simpson D, McMasters KM: Radiofrequency ablation for unresectable hepatic tumors Am J Surg 2001; 182: 552-557. 29-Boweles BJ, Machi J, Limm WM, Severino R, Oishi AJ, Furumoto NL, Wong LL, Oishi RH: Safety and efficacy of radiofrequency thermal ablation in advanced liver tumors. Arch Surg 2001; 136: 864-869.

30-Jiang HC, Liu LX, Piao DX, Xu J, Zheng M,Zhual, Qi SY Zhang WH, Wu LF: Clinical short-term results of radiofrequency ablation in liver cancers. World $J$ Gastroenterology 2002; 8: 624-630.

31-Bleicher RJ, Allegra DP, Nora DT, Wood TF, Foshag LJ, Bilchik AJ: Radiofrequency ablation in 441 complex unresectable liver tumors; lessons learned. Ann Surg Oncol 2003; 10: 52-58.

32-Catalano O, Esposito M, Nunziata A, et al: Multiphase helical CT findings after percutaneous ablation procedures for hepatocellular carcinoma. Abdominal Imaging 2000; 25(6): 607-614.

33-Lim HK, Choi D, Lee WJ, et al: Hepatocellular carcinoma treated with percutaneous radio-frequency ablation: 
Evaluation with follow-up multiphase helical CT. Radiology 2001; 221: 447-454.

34-Goldgerg SN, Gazelle GS, Mueller PR:

Thermal ablation therapy for focal malignancy: A unified approach to underlying principles, techniques, and diagnostic imaging guidance. AJR 2000; 174: 323-331. 